\title{
An image segmentation approach to extract colon lumen through colonic material tagging and hidden Markov random field model for virtual colonoscopy
}

\author{
Lihong $\mathrm{Li}^{\text {*ab }}$, Dongqing Chen ${ }^{\mathrm{b}}$, Sarang Lakare ${ }^{\mathrm{c}}$, Kevin Kreeger ${ }^{\mathrm{c}}$, Ingmar Bitter ${ }^{\mathrm{c}}$, Arie E. Kaufman ${ }^{\mathrm{cb}}$, \\ Mark R. Wax ${ }^{\mathrm{b}}$, Petar M. Djuric ${ }^{\mathrm{a}}$, and Zhengrong Liang ${ }^{\mathrm{bc}}$ \\ Departments of ${ }^{\mathrm{a}}$ Electrical \& Computer Engineering, ${ }^{\mathrm{b}}$ Radiology, and ${ }^{\mathrm{c}}$ Computer Science \\ State University of New York at Stony Brook, Stony Brook, NY 11794
}

\begin{abstract}
Virtual colonoscopy provides a safe, minimal-invasive approach to detect colonic polyps using medical imaging and computer graphics technologies. Residual stool and fluid are problematic for optimal viewing of the colonic mucosa. Electronic cleansing techniques combining bowel preparation, oral contrast agents, and image segmentation were developed to extract the colon lumen from computed tomography (CT) images of the colon. In this paper, we present a new electronic colon cleansing technology, which employs a hidden Markov random filed (MRF) model to integrate the neighborhood information for overcoming the non-uniformity problems within the tagged stool/fluid region. Prior to obtaining CT images, the patient undergoes a bowel preparation. A statistical method for maximum a posterior probability (MAP) was developed to identify the enhanced regions of residual stool/fluid. The method utilizes a hidden MRF Gibbs model to integrate the spatial information into the Expectation Maximization (EM) model-fitting MAP algorithm. The algorithm estimates the model parameters and segments the voxels iteratively in an interleaved manner, converging to a solution where the model parameters and voxel labels are stabilized within a specified criterion. Experimental results are promising.
\end{abstract}

Keywords: virtual colonoscopy, Markov random field, electronic colon cleansing, massive polyp screening

\section{INTRODUCTION}

Colorectal carcinoma currently ranks as the third most common human malignancy and the second leading cause of cancer-related deaths in the United States [3]. The overall risk of developing this disease is approximately 5\% over a lifetime. In 1999 and 2000 there were approximately 130,000 new cases of colorectal cancer and 57,000 deaths each year $[3,18]$. Unfortunately colorectal cancer is often diagnosed at an advanced stage after the patient has developed symptoms. More than $85 \%$ colon cancer arises from adenomatous polyps, which can take 5 to 10 years for malignant transformation [6]. The risk of developing carcinoma from a polyp is directly related to its size: essentially $0 \%$ risk if the polyp is less than $5 \mathrm{~mm}, 1 \%$ risk if size is between 5 to $10 \mathrm{~mm}, 10 \%$ risk with size 10 to $20 \mathrm{~mm}$, and at least $30 \%$ risk with polyps larger than $20 \mathrm{~mm}$ [13]. Survival rates from colon cancer are related directly to the pathologic staging of the disease [1]. As a result, early detection and removal of polyps is the key to prevent colon cancer and can eliminate colon cancer in $90 \%$ of patients.

While currently available optical colonoscopy is the most accurate diagnostic procedure for detecting polyps, it is expensive, invasive, uncomfortable, time consuming and carries a small risk of perforation and death. Patients are usually reluctant for early examination [15]. A minimally invasive, safe, and low-cost method to evaluate the colon for colorectal cancer would be preferred by most patients. Recently, virtual colonoscopy has been developed as an alternative method to evaluate the entire colon for polyps $[5,14,16]$. This new technology utilizes advanced image processing techniques within a computer visualization system for physicians to virtually navigate through a patient's colon model reconstructed from helical abdominal computed tomography (CT) images.

*Correspondence: 1li@clio.rad.sunysb.edu; phone 631 444-2508; fax 631 444-6450; http://www.mipl.rad.sunysb.edu/micl; Laboratory for Imaging Research and Informatics, Department of Radiology, State University of New York, Stony Brook, NY 11794. 
Both optical and virtual colonoscopies require a clean colon lumen, which could be achieved by a physical colon washing such as Golytly. However, this physical bowel washing is often unpleasant to patients. We have developed an alternative approach, termed electronic colon cleansing to virtually clean the colon lumen, therefore, eliminating or minimizing physical bowel washing $[2,11,12,17]$. Based on our preliminary studies, residual stool and fluid are often problematic for virtual colonoscopy. In this paper, we focus on developing a statistical method of maximum a posterior probability (MAP) for an optimal classification. We extended our previous reported MAP-EM (Expectation Maximization) segmentation approach $[9,10,11,12]$ by utilizing a hidden Markov random field (MRF) Gibbs model [19]. In this model, the spatial information of a selected voxel was integrated into the well-established MAP-EM modelfitting algorithm, thus overcoming the non-uniformity problems in the tagged stool/fluid region. Performance of the presented method was evaluated by volunteer and patient data sets.

\section{METHOD AND MATERIALS}

\subsection{Bowel preparation}

Prior to obtaining CT images for virtual colonoscopy, the patient undergoes a one-day bowel preparation of mild laxatives and a low residue diet. In order to enhance residual colonic materials, patients also ingest four $250 \mathrm{cc}$ doses of $2.1 \% \mathrm{w} / \mathrm{v}$ barium sulfate suspension with meals the day before the procedure, as well as two doses of $60 \mathrm{cc}$ of gastroview (diatrizoate meglumine and diatrizoate sodium solution) given during the night before and the morning of the procedure. After the patient's colon is inflated with $\mathrm{CO}_{2}(2-3 \mathrm{~L})$ given through a small bore rectal tube, CT images are obtained using standard virtual colonoscopy parameters, see below.

\subsection{Data acquisition}

A high-speed helical CT scanner (HiSpeed CT/i, GE Medical Systems, Milwaukee, WI) was used to acquire the abdominal images. The imaging protocol parameters are: $120 \mathrm{kVp}, 60-120 \mathrm{~mA}$ (depending on body size), $512 \times 512$ array field-of-view (FOV) (completely covering the body), 1.5-2.0:1 pitch and 3-5 mm collimation (completely covering the entire colon). The scanning time ranges from 30 to 40 seconds, depending upon colon size and acquisition protocol, but obtained within a single breath-hold. Raw (projection) data were reconstructed at $1 \mathrm{~mm}$ intervals with a $512 \times 512$ array size resulting in 300-450 images depending on the height of each patient. Each image element or voxel contains a two-byte integer, reflecting the image density. Each volume image set has a typical size of approximately $210 \mathrm{MB}$. The image data were transferred via Ethernet in DICOM format from the CT scanner to a nearby computer workstation for image processing.

\subsection{Image segmentation}

The algorithm is based on the MAP framework, including a priori information on the neighborhood membership specified by the MRF theory. It iteratively estimates the model parameters through the EM algorithm and segments the voxels by MAP in an interleaved manner, converging to a solution where the model parameters and voxel labels are stabilized within a specified criterion. Implementation of the algorithm consists of two steps: parameter estimation and MAP segmentation.

\subsubsection{Initial parameters}

Figure 1 is a typical histogram obtained from a patient image. This figure reflects the unique characteristics of all abdominal CT images. The three peaks correspond to the air inside the colon, the soft tissue and the muscle, respectively. The enhanced stool, fluid, and bone fall into a small peak beyond the muscle. Most partial volume effects happen in those intensity ranges between the air and the soft tissue and between the muscle and the enhance materials.

Initial parameter estimation for classification can be obtained from the histogram based on thresholds. But it is heuristic. Instead, we proposed a fast online vector quantization segmentation approach $[2,8]$, which has a unique property of independence of initial values. This approach is based on a principal component analysis for the local feature vectors of each voxel. Feature vectors are then classified to a class according to the nearest neighbor rule. The vector quantization 
method supplies us a preliminary segmentation result as the initial parameter estimations. Following that, we focus on segmenting the tagged stool/fluid region using the hidden MRF model to overcome the non-uniformity problems.

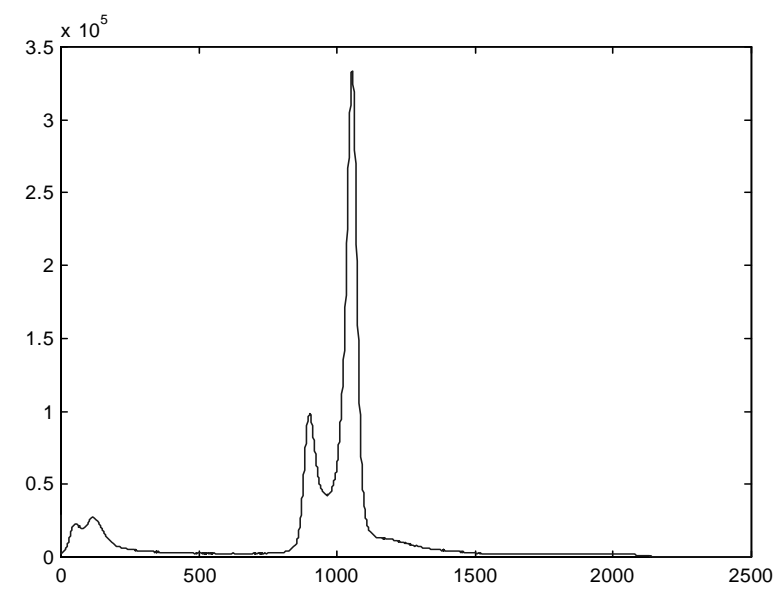

Figure 1: Histogram of a volume CT image from a patient.

\subsubsection{Parameter estimation}

Assume that an image consists of $L$ classes (or tissue types) and each class $l$ is characterized by a Gaussian parameter vector $\theta_{l}\left(\mu_{l}, v_{l}\right)$. Let $p_{l}\left(Y_{i j k} \mid \theta_{l}\right)$ be the probability distribution of voxel $Y_{i j k}$ which is associated with class $l$. The likelihood for each voxel $Y_{i j k}$, falling into $L$ distinct classes, is described by a mixture functional as

$$
p\left(Y_{i j k} \mid X, \Theta\right)=\sum_{l=1}^{L} p\left(l \mid X_{N(i j k)}\right) p_{l}\left(Y_{i j k} \mid \theta_{l}\right)
$$

where $p\left(l \mid X_{N(i j k)}\right)$ is the locally-dependent probability when $X_{i j k}$ is equal to $l$, and $X_{N(i j k)}$ reflects the labels of those nearby voxels.

Assuming a set of initial estimation $\left\{\mu_{l}^{(0)}, v_{l}^{(0)}\right\}_{l=1}^{L}$ and applying the EM algorithm, we have, at each iteration $n$,

$$
\begin{gathered}
Z_{i j k}^{l(n)}=\frac{p^{(n)}\left(l \mid X_{N(i j k)}\right) p_{l}\left(Y_{i j k} \mid \theta_{l}^{(n)}\right)}{\sum_{l=1}^{L} p^{(n)}\left(l \mid X_{N(i j k)}\right) p_{l}\left(Y_{i j k} \mid \theta_{l}^{(n)}\right)} \\
\mu_{l}^{(n+1)}=\frac{\sum_{i, j, k=1}^{I, J, K} Z_{i j k}^{l(n)} Y_{i j k}}{\sum_{i, j, k=1}^{I, J, K} Z_{i j k}^{l(n)}} \\
v_{l}^{(n+1)}=\frac{\sum_{i, j, k=1}^{I, J, K} Z_{i j k}^{l(n)}\left(Y_{i j k}-\mu_{l}^{(n+1)}\right)^{2}}{\sum_{i, j, k=1}^{I, J, K} Z_{i j k}^{l(n)}}
\end{gathered}
$$

where $\mathrm{Z}_{i j k}^{l(n)}$ is the conditional probability that voxel $Y_{i j k}$ belongs to class $l$, which represents the tissue percentages within that voxel. The calculation of tissue percentages $\left\{Z_{i j k}^{l(n)}\right\}$ within each voxel requires determination of the conditional probability $p^{(n)}\left(l \mid X_{(i j k)}\right)$ at the $n$-th iteration. The determination of $p^{(n)}\left(l \mid X_{N(i j k)}\right)$ requires estimation of the class labels $\{l\}$, which can be obtained by the following MAP segmentation method. 


\subsubsection{MAP segmentation method}

A MRF prior is constructed to reflect the neighborhood information $[4,7,10]$. The assignment of labels over the voxel array is performed by the MAP criterion. A Markov random field prior can be constructed to reflect the neighborhood information by

$$
p(X)=\frac{1}{\alpha} \exp \left[-\frac{U(X)}{\beta}\right]
$$

where $\alpha$ is a normalization constant and $\beta$ is constant parameter. The energy function $U(\mathrm{X})$ specifies the degree of penalty imposed on the neighbors, and can be defined, in three dimensions, as

$$
U(\mathrm{X})=\sum_{i=1}^{N}\left\{\sum_{r \in c_{i}^{1}}\left[1-\delta\left(l_{i}-l_{r}\right)\right]+\sum_{s \in c_{i}^{2}}\left[1-\delta\left(l_{i}-l_{s}\right)\right] / \sqrt{2}\right\}
$$

where $\delta(0)=1, \delta(\neq 0)=0$ and $\left\{l_{i}\right\}$ are the voxel labels of the neighbors. The index $r$ runs over the 6 first-order neighbors and $s$ runs over the 12 second-order neighbors.

The segmentation is performed iteratively, where the current voxel is assigned to the corresponding tissue type $l$ at the $(\mathrm{n}+1)$-th iteration by,

$$
\left\{\max _{l \in L}\right\} p_{l}\left(Y_{i j k} \mid \theta_{l}\right) p\left(X_{i j k}^{(n+1)}=\theta_{l} \mid X_{N(i j k)}^{(n)}\right)
$$

\subsection{Extraction of the colon lumen}

Given the mixture segmentation or mixels, we extract a tissue structure based on the similarity of mixels. First, a seed point or voxel is selected by the user for a specific tissue type. The percentage of this specific tissue must be greater than other tissue types inside that voxel. The percentage proportion of all tissue types in the voxel provides a characteristic vector reflecting the specific tissue type. All voxels with similar percentage proportions will be grouped together by utilizing region-growing strategies with modification of adding neighbor mixels into consideration.
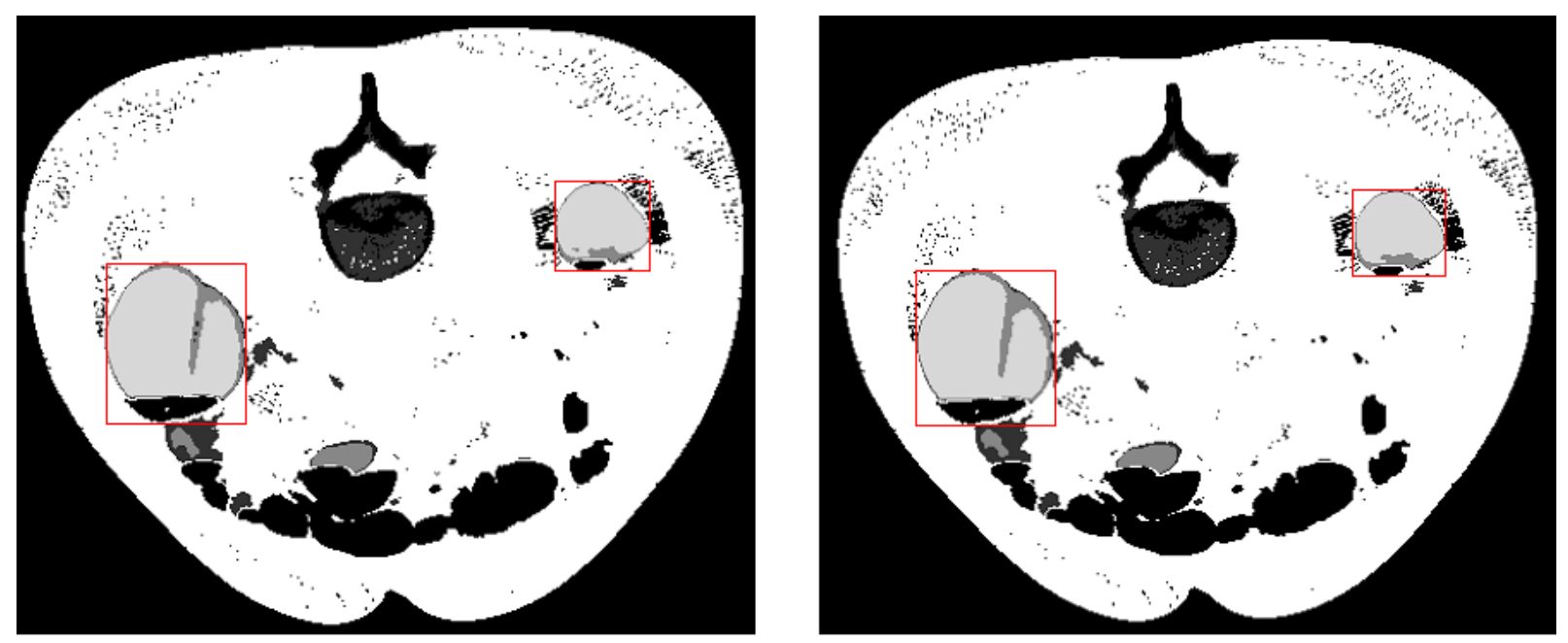

Figure 2: Segmentation results on the tagged region with the standard maximum likelihood EM algorithm (left) and the integration of hidden MRF MAP-EM algorithm (right).

\section{RESULTS}

The new electronic cleansing method has been evaluated by volunteer and patient data sets. Figure 2 demonstrates the segmentation results on the tagged region with the standard maximum likelihood (ML) EM algorithm (left) and the 
integration of hidden MRF MAP-EM algorithm (right). Clearly, the results of the hidden MRF MAP-EM algorithm are much better than those of the standard ML-EM algorithm. Figure 3 demonstrates an overview of the entire extracted colon lumen. Figure 4 is the internal colon view rendered by a virtual colonoscopy system (Viatronix V3D-Colon module, Viatronix Inc., Stony Brook, NY). In addition, the computational performance of the algorithm was also tested. It took approximately 5 minutes for 400 image slices of $512 \times 512$ array size for classifying the tagged region on an AMD $900 \mathrm{MHz}$-based PC system.

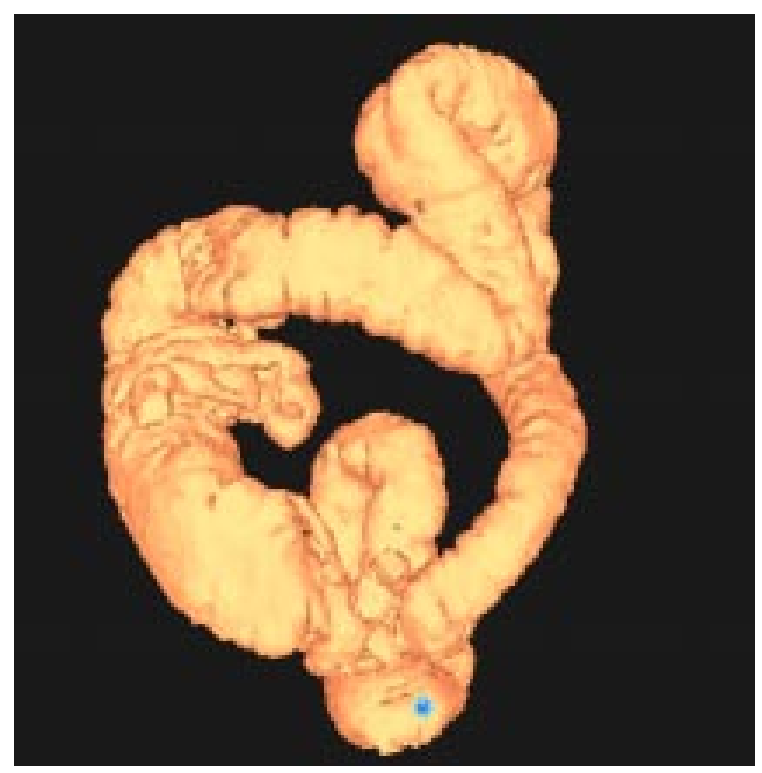

Figure 3: An overview of the entire extracted colon lumen.

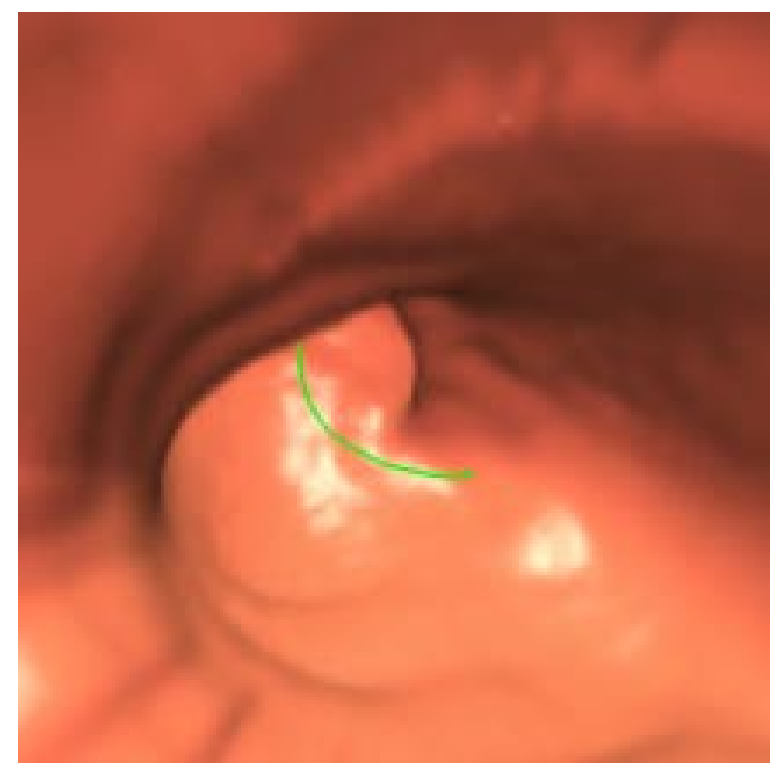

Figure 4: The internal colon view rendered by the virtual colonoscopy system.

\section{DISCUSSION AND CONCLUSIONS}

We have presented an electronic colon cleansing technology based on a hidden MRF model and MAP-EM algorithm for extracting the colon lumen from abdominal CT images. The algorithm has been tested on a virtual colonoscopy system that enables real-time volume rendering with interactive and planned endoscopic navigation through the colon to detect polyps. This method can detect colon polyps as part of a colorectal cancer.

In this approach, the algorithm models the tagged materials and colon object by an isotropic Markov random field. Unlike the non-MRF approaches, our MRF-based spatial information has been integrated to the EM algorithm to estimate model parameters and segment voxels simultaneously. The use of a hidden MRF model overcomes the nonuniformity problems in the tagged fluid/stool regions, which are the major obstacle for virtual colonoscopy without the conventional physical colon washing procedure. By eliminating or minimizing this physical bowel preparation with stool/fluid tagging and image segmentation, virtual colonoscopy will be more tolerated and accepted by patients.

\section{ACKNOWLEDGEMENTS}

This work was supported by NIH Grant No. CA82402 of the National Cancer Institute and partially by Viatronix, Inc. The volunteer and patient data sets were provided by the University Hospital of the State University of New York at Stony Brook. 


\section{REFERENCES}

1. T. Byers, B. Levin, D. Rothenberger, et al., "American Cancer Society guidelines for screening and surveillance for early detection of colorectal polyps and cancer: update 1997," CA Cancer J Clin, vol. 47, pp. 154-160, 1997.

2. D. Chen, Z. Liang, M. R. Wax, L. Li, B. Li, and A. E. Kaufman, "A novel approach to extract colon lumen from CT images for virtual colonoscopy," IEEE Trans. Medical Imaging, vol. 19, pp. 1220-1226, 2000.

3. R. T. Greenlee, T. Murray, S. Bolden, P. A. Wingo, "Cancer statistics," Ca Cancer J Clin, vol. 50, pp. 7-33, 2000.

4. K. Held, E. R. Kops, B. J. Krause, W. M. Wells, and R. Kikinis, "Markov random field segmentation of brain MR images," IEEE Trans. Medical Imaging, vol. 16, pp. 878-886, 1997.

5. L. Hong, A. Kaufman, Y.-C. Wei, A. Viswambharan, M. Wax, and Z. Liang, "3-D virtual colonoscopy," Proc. Biomedical Visualization, M. Loew and N. Gershon, Eds., Altanta, GA, pp. 26-33, 1995.

6. R. Koretz, "Malignant polyps: Are they sheep in wolves' clothing?” Annals of Internal Med., 118, pp. 63-68, 1993.

7. R. Leahy, T. Hebert, and R. Lee, "Applications of Markov random fields in medical imaging," Information Processing in Medical Imaging, pp. 1-14, 1991.

8. L. Li, D. Chen, H. Lu, and Z. Liang, "Segmentation of MR brain images: a self-adaptive online vector quantization approach," SPIE Medical Imaging, vol. 4322, pp. 1431-1438, 2001.

9. Z. Liang, R. J. Jaszczak, and R. E. Coleman, "Parameter estimation of finite mixtures using the EM algorithm and information criteria with application to medical image processing," IEEE Trans. Nuclear Science, vol. 39, pp. 1126$1133,1992$.

10. Z. Liang, J. R. MacFall, and D. P. Harrington, "Parameter estimation and tissue segmentation from multispectral MR images," IEEE Trans. Medical Imaging, vol. 13, pp. 441-449, 1994.

11. Z. Liang, D. Chen, R. Chiou, B. Li, A. Kaufman, and M. Wax, "On segmentation of colon lumen for virtual colonoscopy,” SPIE Medical Imaging, vol. 3660, pp. 270-278, 1999.

12. Z. Liang, F. Yang, M. Wax, J. Li, J. You, A. Kaufman, L. Hong, H. Li, and A. Viswambharan, "Inclusion of $a$ priori information in segmentation of colon lumen for virtual colonoscopy," Conf Record IEEE NSS-MIC, 1997.

13. J. S. Mandel, J. H. Bond, T. R. Church, and D. Snover, "Reducing mortality from colorectal cancer by screening with FOBT," New Engl J Medicine, vol. 328, pp. 1365-1371, 1993.

14. E. McFarland, J. Brink, J. Loh, G. Wang, V. Argiro, D. Balfe, J. Heiken, and M. Vannier, "Visualization of colorectal polyps with spiral CT colography: Evaluation of processing parameters with perspective volume rendering," Radiology, vol. 205, pp. 701-707, 1997.

15. P. Orsoni, S. Berdah, C. Verrier, et al. "Colonic perforation due to colonoscopy: a retrospective study of 48 cases," Endocsopy, vol. 29, pp. 160-164, 1997.

16. D. J. Vining, D. Gelfand, R. Bechtold, E. Scharling, E. F. Grishaw, and T. Shifirin, "Technical feasibility of colon imaging with helical CT and virtual reality," Ann. Meeting Amer. Roentgen Ray. Soc., pp. 104, 1994.

17. M. Wax, Z. Liang, D. Chen, B. Li, R. Chiou, A. Kaufman, and A. Viswambharan, "Electronic colon cleansing for virtual colonoscopy," $1^{\text {st }}$ Intl. Conference on Virtual Colonoscopy, Boston, MA, 1998.

18. S. J. Winawer, R. H. Fletcher, L. Miller, et al., "Colorectal cancer screening: clinical guidelines and rational," Gastroenterology, vol. 112, pp. 594-642, 1997.

19. Y. Zhang, M. Brady, and S. Smith, "Segmentation of brain MR images through a hidden Markov random field model and the expectation-maximization algorithm," IEEE Trans. Medical Imaging, vol. 20, pp. 45-57, 2001. 